\title{
Analysis of the W 44 Supernova Remnant and its surroundings with Fermi-LAT and MAGIC
}

\author{
Di Tria R., ${ }^{a}$ Di Venere L., ${ }^{a, *}$ Giordano F., ${ }^{a}$ Green D., ${ }^{b}$ Hahn A., ${ }^{b}$ Pantaleo F.R. ${ }^{a}$ and \\ Strzys M. $^{c}$ on behalf of the Fermi-LAT and MAGIC Collaborations ${ }^{\dagger}$ \\ a Dipartimento Interateneo di Fisica dell'Università e del Politecnico di Bari and INFN Sezione di Bari, via \\ Orabona 4, 70125, Bari, Italy \\ ${ }^{b}$ Max-Planck-Institut for Physics, Föhringer Ring 6, 80805, München, Germany \\ ${ }^{c}$ Institute for Cosmic Ray Research 1035 (ICRR), The University of Tokyo, Kashiwa, 277-8582 Chiba, \\ Japan \\ E-mail: leonardo.divenere@ba.infn.it
}

The well-known supernova remnant (SNR) W 44 is observed in high-energy gamma rays and widely studied to investigate cosmic ray (CR) acceleration. Several analyses of the W 44 surroundings showed the presence of gamma-ray emission offset from the radio SNR shell. This emission is thought to originate from escaped high-energy CRs. We present a detailed analysis of the W 44 region as seen by Fermi-LAT, focusing on the spatial and spectral characteristics of both W 44 SNR and its surroundings. The spatial analysis was limited to energies above $1 \mathrm{GeV}$ in order to exploit the improved angular resolution of the instrument, deriving a detailed description of the region morphology. Observations of the north-western region of $\mathrm{W} 44$, also known as SRC-1 from previous works, were conducted with the MAGIC telescopes in the very high-energy gamma-ray band. We analysed MAGIC data exploiting the spatial information derived with the Fermi-LAT analysis at GeV energies. Here we show the results of both analyses and the combined Fermi-LAT and MAGIC spectra, thus obtaining constraining information on the diffusion of the escaped CRs.

$37^{\text {th }}$ International Cosmic Ray Conference (ICRC 2021)

July 12 th - 23rd, 2021

Online - Berlin, Germany

\footnotetext{
${ }^{*}$ Presenter

${ }^{\dagger}$ a complete list of the MAGIC Collaboration authors can be found at the end of the proceedings
} 


\section{Introduction}

Supernova Remnants (SNRs) play a central role in the study of Cosmic rays (CRs) and in particular to explain the origin of Galactic CRs. W 44 is one of the most luminous and studied SNR in the gamma-ray energy band. AGILE and Fermi-LAT reported its emission with strong evidence of hadronic interaction of accelerated cosmic rays with the dense environment $[1,2]$. It is a middle-aged SNR $\left(\sim 2 \cdot 10^{4} y r\right)$, which is the optimal age to study the escaping of cosmic rays from the acceleration region of the SNR. In particular, the softening of the spectrum observed around tens of $\mathrm{GeV}$, already suggested a possible escape of high energy cosmic rays. Studies conducted on the surrounding region revealed an emission above the one expected from the cosmic ray sea. Uchiyama et al. [2] discovered two extended regions emitting at $\mathrm{GeV}$ energies close to the $\mathrm{W} 44$ shell, which are thought to derive from escaped cosmic rays interacting with the dense medium. Peron et al. [3] recently confirmed this hypothesis.

In this contribution, we investigate the $\mathrm{W} 44$ region from $\mathrm{GeV}$ to $\mathrm{TeV}$ energies with Fermi-LAT and MAGIC data. In particular, we analysed the whole W 44 region with Fermi-LAT at energies above $1 \mathrm{GeV}$, both spatially and spectrally. Afterwards, we analysed the North-Western region of W 44 (also known as SRC-1 [2]) with MAGIC telescopes at energies above an analysis threshold of $130 \mathrm{GeV}$.

\section{Fermi-LAT analysis}

The Large Area Telescope (LAT) instrument onboard the Fermi Gamma-Ray Space Telescope satellite is a pair-conversion telescope with a precision converter-tracker and calorimeter that detects gamma rays from tens of $\mathrm{MeV}$ to hundreds of $\mathrm{GeV}$.

We selected almost 12 years of P8_R3 data from a Region of Interest (RoI) of $15^{\circ}$ centered on the $\mathrm{W} 44$ position (GLON: $34.65^{\circ}$, GLAT: $-0.38^{\circ}$ ) in the energy range from $1 \mathrm{GeV}$ to $2 \mathrm{TeV}$. We selected SOURCE class events with a maximum zenith angle of $105^{\circ}$ to get rid of the Earth's limb contamination.

We performed a binned likelihood analysis in a squared region inscribed in the RoI selected. Data were binned in energy adopting 8 bins/decade. In order to increase the Fermi-LAT sensitivity, we divided the dataset according to the PSF event types available in the Pass 8 reprocessing of Fermi-LAT data. The event types available go from PSF0 to PSF 3 with increasingly better angular reconstruction. The corresponding set of IRFs, P8R3_SOURCE_V3, was used to perform the fitting procedure. The RoI was described including in the model all the known sources from the 4FGLDR2 catalog [4] within $20^{\circ}$ from the RoI center and the Galactic and isotropic diffuse background models (gll_iem_v07,iso_P8R3_SOURCE_V3_v1'1). Among these, the normalization parameters of the diffuse components and of sources having a significance larger than 20 were fitted, while other parameters were kept fixed at the catalog values. The analysis was conducted using the fermitools (v2.0.8) and the fermipy (v1.0.1) packages.

A detailed morphological analysis of the W 44 region was conducted. For this scope, we first removed all known sources within $1^{\circ}$ from the RoI center, except for the W 44 . Afterwards, a

\footnotetext{
${ }^{1}$ See here https://fermi.gsfc.nasa.gov/ssc/data/access/lat/BackgroundModels.html for more details.
} 
source-find algorithm was applied to detect new point-like sources within $1^{\circ}$ from the center of the RoI and having a significance above $5 \sigma$. Each newly found source was subjected to an extension fitting procedure in order to determine whether an extended spatial model is more suitable. A disk model was used instead of the point-like model, and the radius of the disk was fitted to the data. A source is considered extended if $T S_{\text {ext }}>25^{2}$. Finally, the new sources underwent also a curvature fitting procedure in order to determine whether their spectra were characterized by a certain degree of curvature or not. In the reference model $M_{0}$, the source under investigation was described with a power-law spectrum $\frac{d N}{d E}=N_{0}\left(\frac{E}{E_{0}}\right)^{-\gamma}$. For the alternative model the Log-Parabola spectrum ${ }^{3}$ was considered. The $T S_{\text {curv }}$ was calculated as $2\left(\ln L_{1}-\ln L_{0}\right)$ and the source was considered "curved" if $T S_{\text {curv }}>20$.

The procedure previously described was repeated for different spatial template describing the morphology of the $\mathrm{W} 44 \mathrm{SNR}$. The choice of the best configuration was made evaluating the value of the Akaike Information Criterion (AIC)[5] for each analysis result, defined as AIC $=2 k-2 \ln L$, where $k$ is the number of parameters of the model. The best model is the one for which the AIC is minimized. Since the variation in the number of parameters depends essentially on what happens within $1^{\circ}$ from the center of the ROI, we considered only the sources in this restricted region for the calculation of the $k$.

The spatial templates considered for the description of the morphology of the remnant were: the 4FGL catalog template, derived in [6], a radio $(1420 \mathrm{MHz})$ template derived from the THOR survey [7] and a full-ellipse template. The catalog template and the full-ellipse template were also divided along the major axis. The two half-ellipses were fitted as independent sources (doubling the number of free parameters).

Regarding the elliptical model, the dimension and orientation of the full-ellipse template were derived with a dedicated analysis, by fitting templates with varying inclination angles with $10^{\circ}$-steps, semi-major and semi-minor axes with $0.04^{\circ}$-steps. The AIC criterion was used to determine the best elliptical template. The minimum AIC value was obtained for $115^{\circ}$ of inclination angle, $a=0.38^{\circ}$, $b=0.2^{\circ}$. A finer step of $0.01^{\circ}$ was used for the ellipse semi-axes, fixing the inclination angle to $115^{\circ}$. The log-likelihood resulted maximized and the AIC minimized (Fig. 1$)$ for $\left[a=(0.41 \pm 0.01)^{\circ}\right.$, $\left.b=(0.23 \pm 0.01)^{\circ}\right]$.

Table1 shows the log-likelihood and AIC values for all the models adopted, showing that the best result is obtained with the radio template. Fig. 2 shows a deviation probability map ${ }^{4}$ obtained with the best model. The red contours represent the W 44 morphology adopted while the red crosses and circles show the sources added and fitted in this analysis. The black crosses and circles represent all the sources from the 4FGL-DR2 catalog. In particular, two moderately extended sources located at the opposite edges along the major axis of the SNR were detected, which are believed to be the North-West (NW) and South-East (SE) sources already observed in previous works [2, 3]. As a consequence of the extension fitting procedure, each of them was spatially modelled with a disk.

\footnotetext{
${ }^{2} T S_{\text {ext }}$ is defined as twice the log-likelihood difference between a model $H 1$ with an extended model and a null hypothesis $\mathrm{HO}$ based on a point-like source.

${ }^{3}$ https://fermi.gsfc.nasa.gov/ssc/data/analysis/scitools/source_models.html

${ }^{4}$ The deviation probability map shows the data/model agreement and is sensitive to both positive and negative fluctuations. See here for more details https://indico.cern.ch/event/1010947/contributions/4278096/ attachments/2228212/3774978/bruel_FermiSymposium2021_PSmap_v4.pdf
} 

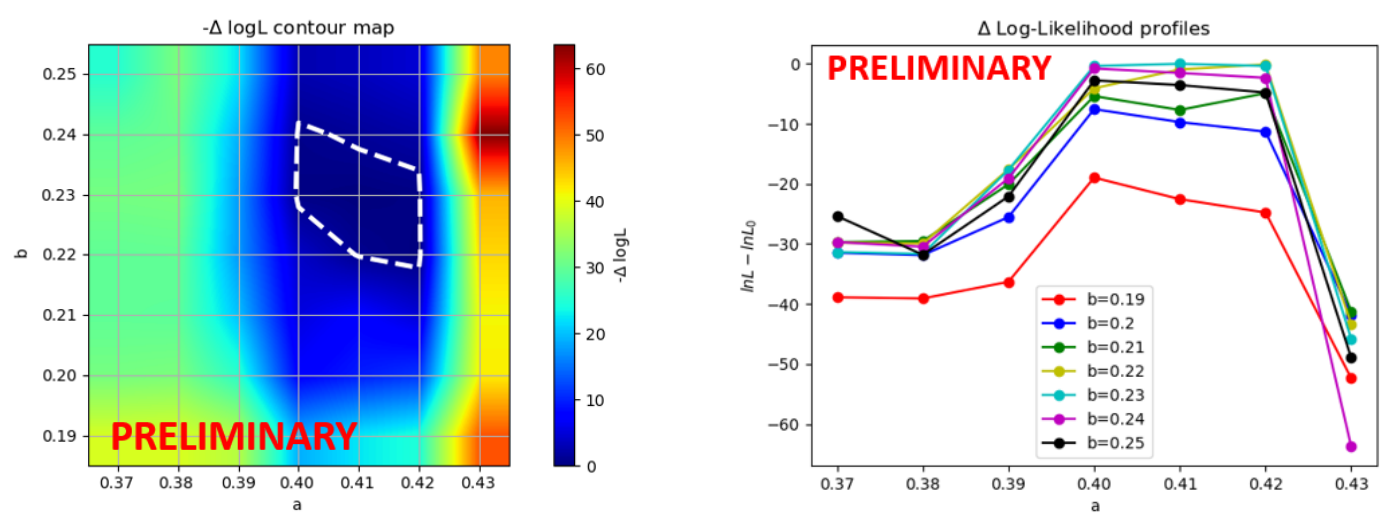

Figure 1: Left: 2-dimensional Log-Likelihood profile obtained with full elliptical template with an inclination angle of $115^{\circ}$. The white contour represent the $68 \%$ confidence level border. Right: $\Delta$ log-likelihood $\left(\ln L-\ln L_{\text {max }}\right)$ profile as a function of the semi-major axes $a$ of the ellipse for fixed values of the semi-minor axis $b$.

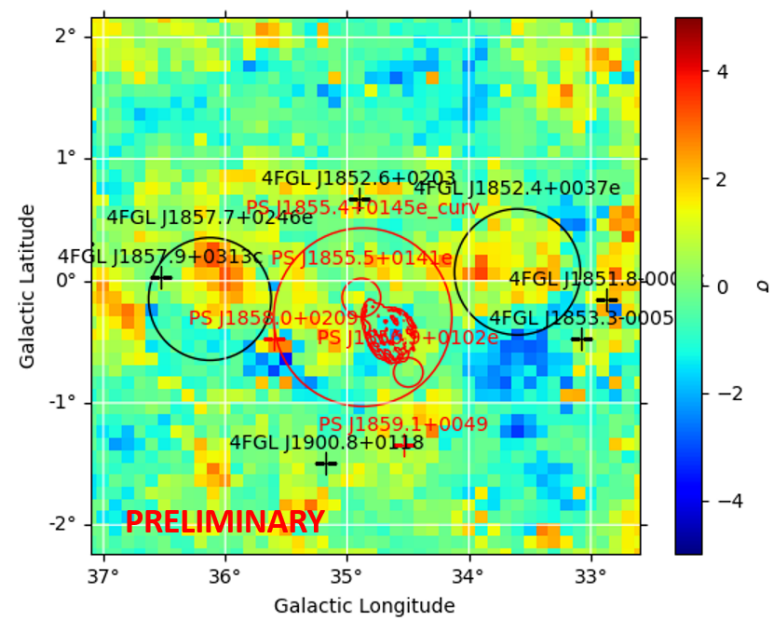

Figure 2: Deviation probability map, or PS map, of the W 44 surroundings with the best model obtained from the likelihood analysis. Red contours represent the W 44 radio template adopted in the analysis. The additional red crosses and circles show the newly sources added in this analysis, while the black ones correspond to sources in the 4FGL-DR2 Fermi-LAT catalog. The radii of the circles represent the $r_{68}$ of the extended sources modelled with a disk shape.

In addition to the two small disks, a large central extended source needs to be added to the model to describe a residual diffuse $\gamma$-ray emission originating in the surroundings of W 44. Table 2 shows the position and radius of the "diffuse" disk as well as those of the two small NW and SE extended sources.

The morphology of this diffuse emission was investigated in detail. In order to find possible associations with gas complexes, we studied ${ }^{12} \mathrm{CO}$ and ${ }^{13} \mathrm{CO}$ data from the NRO FUGIN survey obtained with the Nobeyama $45 \mathrm{~m}$-telescope [8] in a region within $\sim 1.5^{\circ}$ from the SNR.Nine velocity intervals were chosen from the emission profiles of the gas in correspondence of the large 


$\begin{array}{lcccc}\text { Template (W 44) } & \operatorname{lnL} & \mathbf{k} \text { (d.o.f.) } & \text { AIC } & \Delta_{\text {AIC }} \\ \text { 4FGL } & 57702 & 18 & -115368 & 289 \\ \text { 4FGL divided } & 57755 & 25 & -115460 & 197 \\ \text { Full ellipse } & 57743 & 18 & -115450 & 207 \\ \text { Full ellipse divided } & 57770 & 20 & -115501 & 156 \\ \text { Radio (1420 MHz) } & 57856 & 27 & -115657 & 0\end{array}$

Table 1: Results for 4FGL, full ellipse and radio templates. The $\Delta_{A I C}$ is calculated with respect to the minimum AIC, corresponding to the 'radio' template.

$\begin{array}{lccc}\text { Sources } & \text { RA }(\mathbf{d e g}) & \text { DEC }(\mathbf{d e g}) & r_{68}(\mathbf{d e g}) \\ \text { NW source } & 283.87 \pm 0.01 & 1.67 \pm 0.01 & 0.16 \pm 0.01 \\ \text { SE source } & 284.24 \pm 0.01 & 1.05 \pm 0.01 & 0.12 \pm 0.01 \\ \text { DIFFUSE disk } & 284.01 \pm 0.02 & 1.59 \pm 0.02 & 0.73 \pm 0.02\end{array}$

Table 2: Fitted position and radius of the three extended sources of Fig. 2. $r_{68}$ represents the $68 \%$ containment radius of the best-fit spatial model.

disk, and in correspondence of the NW and SE disks. The velocity intervals corresponded to peaks of the gas profile within each of these regions. For each case we derived a template and repeated the likelihood fitting procedure for Fermi-LAT data, adopting the radio template for the W 44 . The other sources in the RoI and in particular the NW and SE sources were refitted both spatially and spectrally in each case. The AIC value was used to obtain the best configuration. Among the CO templates derived, the one that provided the best results corresponds to the velocity interval (38.6 - 49.8) km/s, which is shown in Fig. 3. However, the large disk derived previously (and reported in table 2) still provided the best description of the RoI, with a $\triangle \mathrm{AIC}=10.6$ in favour of the disk hypothesis. Therefore, this latter model was used as reference spatial model also for the MAGIC analysis.

It is important to note that when using the CO template instead of the large disk to model the large diffuse background, the spatial and spectral parameters of both the NW and SE sources did not change significantly, being compatible with the results in table 2 within the statistical uncertainties.

Finally, a spectral analysis was conducted on the whole region. A curvature test was performed using a Log-Parabola spectrum, as described at the beginning of this section. The Spectral Energy Distribution (SED) of each source was calculated in the energy range from $1 \mathrm{GeV}$ to $2 \mathrm{TeV}$, assuming a binning of 8 bins/decade for energies $\leq 30 \mathrm{GeV}$ and 4 bins/decade for energies $>30 \mathrm{GeV}$. Fig. 4 shows the SEDs of the W 44 and of the three extended sources in the W 44 surroundings.

\section{MAGIC analysis}

The Major Atmospheric Gamma Imaging Cherenkov (MAGIC) telescopes are a system of two IACTs located on the Canary island of La Palma at an altitude of $2200 \mathrm{~m}$ above sea level [9]. MAGIC observed the W 44 region between April 2013 and August 2014 for $173.7 \mathrm{~h}$ after quality cuts at zenith angles between $25^{\circ}-45^{\circ}$. The MAGIC observations were centered on the coordinates of NW source from Uchiyama et al. [2] using the standard wobble distance of $0.4^{\circ}$. The low-level 


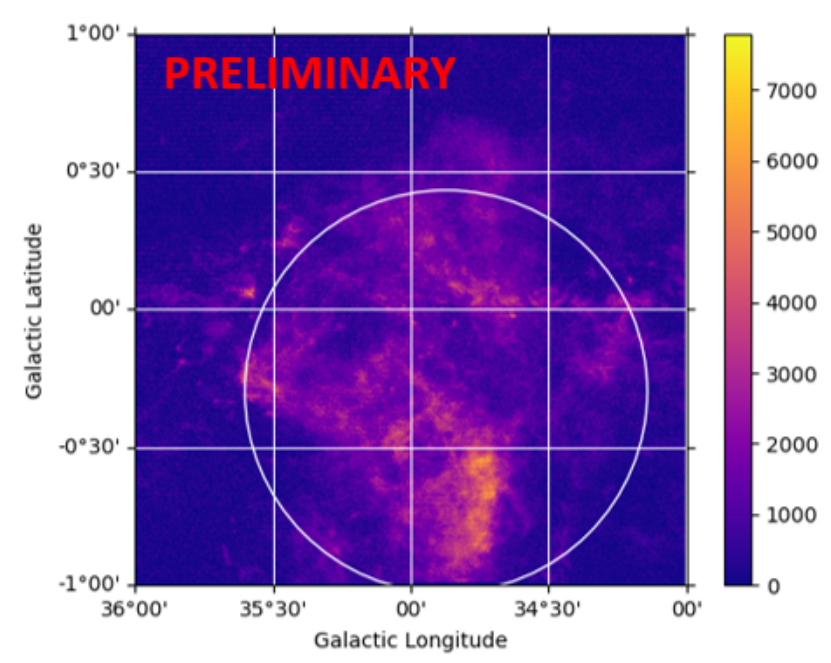

Figure 3: CO template derived from NRO FUGIN survey in the velocity interval $(38.6,49.8) \mathrm{km} / \mathrm{s}$. The template is the sum of both ${ }^{12} \mathrm{CO}$ and ${ }^{13} \mathrm{CO}$ data. For comparison, the white circle represents the diffuse disk derived from the Fermi-LAT analysis.

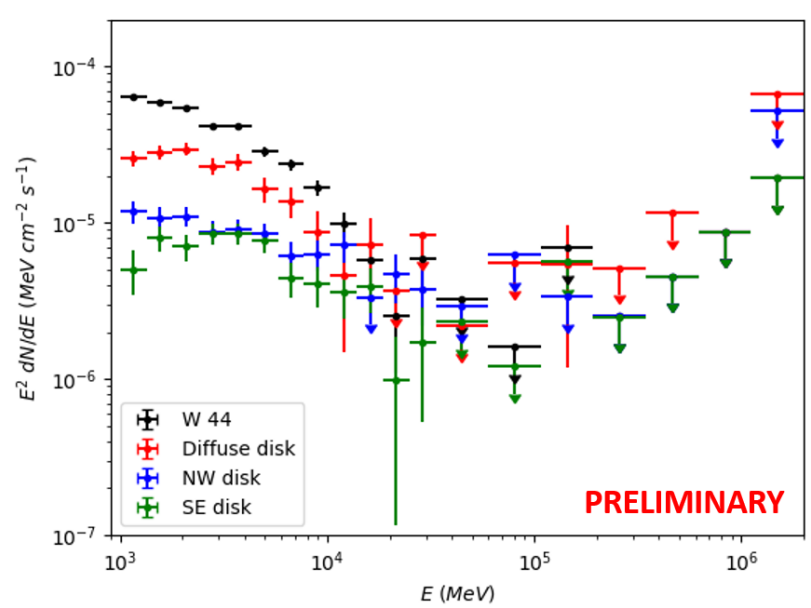

Figure 4: Spectral Energy Distribution of the W 44 SNR, NW, SE small disks and large diffuse disks derived with Fermi-LAT data.

analysis was performed with the MAGIC Analysis and Reconstruction Software (MARS) [10]. We used the spatial likelihood analysis package SkyPrism [11] for our subsequent high-level analysis. An estimation of the systematic uncertainties of the MAGIC telescopes and the low and high-level analysis pipelines can be found in Aleksić et al. [12] and Vovk et al. [11]. Uncertainties are directly calculated from the log-likelihood deviation from the obtained best fit.

From the wobble observations we constructed the background camera exposure model using an Exclusion Map. We excluded the known sources in our field of view (FoV). Namely HESS J1857+026 [13] using an exclusion zone radius of $0.45^{\circ}$ which is large enough to enclose the two known high energy sources MAGIC J1857.2+0263 (RA: $18^{\mathrm{h}} 57^{\mathrm{m}} 13.0^{\mathrm{s}}$ Dec: $02^{\circ} 37^{\prime} 31^{\prime \prime}$ ), 


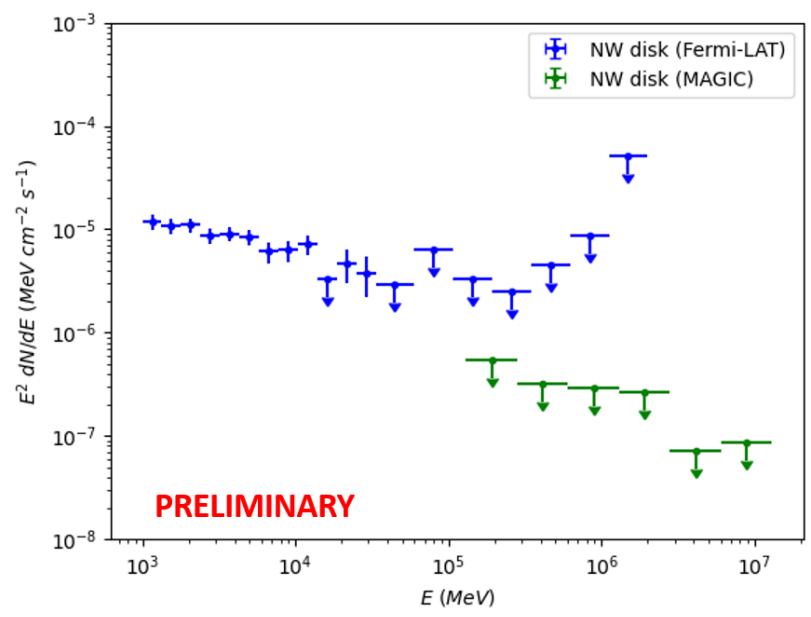

Figure 5: Spectral Energy Distribution of the NW source obtained combining the Fermi-LAT (blue) and MAGIC (green) data. The light blue lines represent the gamma-ray emission in the hypothesis of CRs escaped from W 44 and illuminating a nearby cloud for three values of the diffusion coefficient, as derived in [2].

MAGIC J1857.6+0297 (RA: $18^{\mathrm{h}} 57^{\mathrm{m}} 35.6^{\mathrm{s}}$ Dec: $02^{\circ} 58^{\prime} 02^{\prime \prime}$ ) [14]. We also excluded HESS J1858+020 [13] with a radius of $0.17^{\circ}$, NW and SE with their position and extension from the Fermi-LAT analysis above. The centers of the exclusion regions around HESS J1857+026 and HESS J1858+020 where set to the respective maximum in our sky map. Due to the curved spectra of W 44 and the large background components theses sources do not need to be included in the analysis of MAGIC data, being their flux negligible at energies above $100 \mathrm{GeV}$.

A signal was searched in the region of the NW disk, taking the center and radius derived in the previous Fermi-LAT analysis. No significant detection was obtained with MAGIC. We therefore calculate 95\% CL upper limits in each of our energy bins, which are shown in Fig. 5, together with Fermi-LAT data related to the NW source.

\section{Summary}

We report here on the observations of the W 44 SNR and its surroundings with Fermi-LAT and MAGIC data. Previous works already reported the detection of this SNR and of localized $\mathrm{GeV}$ emissions in its surroundings with Fermi-LAT. Observations were conducted with the MAGIC telescopes centered on one of these surrounding emissions, namely the source known as SRC-1 or NW emission.

A detailed morphological analysis of the whole $\mathrm{W} 44$ region was conducted in this work with the Fermi-LAT data above $1 \mathrm{GeV}$, deriving both spatial and spectral information. The spatial parameters of the NW emission were used to analyse MAGIC data. No detection at energies above $100 \mathrm{GeV}$ was found with these observations and upper limits on the differential flux were derived from MAGIC data. 


\section{Acknowledgments}

The Fermi-LAT Collaboration acknowledges support for LAT development, operation and data analysis from NASA and DOE (United States), CEA/Irfu and IN2P3/CNRS (France), ASI and INFN (Italy), MEXT, KEK, and JAXA (Japan), and the K.A. Wallenberg Foundation, the Swedish Research Council and the National Space Board (Sweden). Science analysis support in the operations phase from INAF (Italy) and CNES (France) is also gratefully acknowledged. This work performed in part under DOE Contract DE-AC02-76SF00515. We acknowledge the support from the agencies and organizations listed here: https://magic.mpp.mpg.de/acknowledgments_ ICRC2021.

\section{References}

[1] Giuliani, et al. 2011, The Astrophysical Journal, 742, L30, publisher: American Astronomical Society

[2] Uchiyama, et al. 2012, The Astrophysical Journal Letters, 749, L35, arXiv: 1203.3234

[3] Peron, G., Aharonian, F., Casanova, S., Zanin, R., \& Romoli, C. 2020, The Astrophysical Journal, 896, L23, publisher: American Astronomical Society

[4] Abdollahi, S., Acero, F., Ackermann, M., et al. 2020, The Astrophysical Journal Supplement Series, 247, 33, publisher: American Astronomical Society

[5] Akaike H., 1998 "A New Look at the Statistical Model Identification", pages 215-222, Springer New York.

[6] Abdo, A. A., et al. Science, 327, February 2010

[7] Beuther, H., Bihr, S., Rugel, M, et al. 2016, Astronomy Astrophysics, 595, A32

[8] Umemoto, T., et al. (2017), Publications of the Astronomical Society of Japan, 69(5), URL: http://dx.doi.org/10.1093/pasj/psx061,doi:10.1093/pasj/psx061.

[9] Aleksić, et al. 2016a, Astroparticle Physics, 72, 61

[10] Zanin, R. 2013, in Proceedings, 33rd International Cosmic Ray Conference (ICRC2013): Rio de Janeiro, Brazil, July 2-9, 2013, 0773

[11] Vovk, I., Strzys, M., \& Fruck, C. 2018, Astronomy \& Astrophysics, 619, A7, publisher: EDP Sciences

[12] Aleksić, et al. 2016b, Astroparticle Physics, 72, 76

[13] Abdalla, et al. 2018, Astronomy \& Astrophysics, 612, A1, publisher: EDP Sciences

[14] Aleksić, et al. 2014, Astronomy \& Astrophysics, 571, A96 


\section{The MAGIC Collaboration}

V. A. Acciari ${ }^{1}$, S. Ansoldi ${ }^{2,41}$, L. A. Antonelli ${ }^{3}$, A. Arbet Engels $^{4}$, M. Artero ${ }^{5}$, K. Asano $^{6}$, D. Baack ${ }^{7}$, A. Babić ${ }^{8}$, A. Baquero ${ }^{9}$, U. Barres de Almeida ${ }^{10}$, J. A. Barrio ${ }^{9}$, I. Batković ${ }^{11}$, J. Becerra González ${ }^{1}$, W. Bednarek ${ }^{12}$, L. Bellizzi ${ }^{13}$, E. Bernardini ${ }^{14}$, M. Bernardos ${ }^{11}$, A. Berti ${ }^{15}$, J. Besenrieder ${ }^{15}$, W. Bhattacharyya ${ }^{14}$, C. Bigongiari ${ }^{3}$, A. Biland ${ }^{4}$, O. Blanch ${ }^{5}$, H. Bökenkamp ${ }^{7}$, G. Bonnoli ${ }^{16}$, Ž. Bošnjak $^{8}$, G. Busetto ${ }^{11}$, R. Carosi ${ }^{17}$, G. Ceribella ${ }^{15}$, M. Cerruti ${ }^{18}$, Y. Chai ${ }^{15}$, A. Chilingarian ${ }^{19}$, S. Cikota $^{8}$, S. M. Colak ${ }^{5}$, E. Colombo ${ }^{1}$, J. L. Contreras ${ }^{9}$, J. Cortina ${ }^{20}$, S. Covino ${ }^{3}$, G. D’Amico ${ }^{15,42}$, V. D’Elia ${ }^{3}$, P. Da Vela ${ }^{17,43}$, F. Dazzi ${ }^{3}$, A. De Angelis ${ }^{11}$, B. De Lotto ${ }^{2}$, M. Delfino ${ }^{5,44}$, J. Delgado ${ }^{5,44}$, C. Delgado Mendez ${ }^{20}$, D. Depaoli ${ }^{21}$, F. Di Pierro ${ }^{21}$, L. Di Venere ${ }^{22}$, E. Do Souto Espiñeira ${ }^{5}$, D. Dominis Prester $^{23}$, A. Donini ${ }^{2}$, D. Dorner ${ }^{24}$, M. Doro ${ }^{11}$, D. Elsaesser ${ }^{7}$, V. Fallah Ramazani ${ }^{25,45}$, A. Fattorini ${ }^{7}$, M. V. Fonseca ${ }^{9}$, L. Font ${ }^{26}$, C. Fruck ${ }^{15}$, S. Fukami ${ }^{6}$, Y. Fukazawa ${ }^{27}$, R. J. García López ${ }^{1}$, M. Garczarczyk ${ }^{14}$, S. Gasparyan ${ }^{28}$, M. Gaug ${ }^{26}$, N. Giglietto ${ }^{22}$, F. Giordano ${ }^{22}$, P. Gliwny ${ }^{12}$, N. Godinović ${ }^{29}$, J. G. Green ${ }^{3}$, D. Green ${ }^{15}$, D. Hadasch ${ }^{6}$, A. Hahn ${ }^{15}$, L. Heckmann ${ }^{15}$, J. Herrera ${ }^{1}$, J. Hoang ${ }^{9,46}$, D. Hrupec ${ }^{30}$, M. Hütten ${ }^{15}$, T. Inada ${ }^{6}$, K. Ishio ${ }^{12}$, Y. Iwamura ${ }^{6}$, I. Jiménez Martínez ${ }^{20}$, J. Jormanainen ${ }^{25}$, L. Jouvin ${ }^{5}$, M. Karjalainen ${ }^{1}$, D. Kerszberg ${ }^{5}$, Y. Kobayashi ${ }^{6}$, H. Kubo ${ }^{31}$, J. Kushida ${ }^{32}$, A. Lamastra ${ }^{3}$, D. Lelas ${ }^{29}$, F. Leone ${ }^{3}$, E. Lindfors ${ }^{25}$, L. Linhoff ${ }^{7}$, S. Lombardi ${ }^{3}$, F. Longo ${ }^{2,47}$, R. López-Coto ${ }^{11}$, M. López-Moya ${ }^{9}$, A. López-Oramas ${ }^{1}$, S. Loporchio ${ }^{22}$, B. Machado de Oliveira Fraga $^{10}$, C. Maggio ${ }^{26}$, P. Majumdar ${ }^{33}$, M. Makariev ${ }^{34}$, M. Mallamaci ${ }^{11}$, G. Maneva ${ }^{34}$, M. Manganaro ${ }^{23}$, K. Mannheim ${ }^{24}$, L. Maraschi ${ }^{3}$, M. Mariotti ${ }^{11}$, M. Martínez ${ }^{5}$, D. Mazin ${ }^{6,15}$, S. Menchiari ${ }^{13}$, S. Mender ${ }^{7}$, S. Mićanović23, D. Miceli ${ }^{2,49}$, T. Miener ${ }^{9}$, J. M. Miranda ${ }^{13}$, R. Mirzoyan ${ }^{15}$, E. Molina ${ }^{18}$, A. Moralejo ${ }^{5}$, D. Morcuende ${ }^{9}$, V. Moreno ${ }^{26}$, E. Moretti ${ }^{5}$, T. Nakamori ${ }^{35}$, L. Nava ${ }^{3}$, V. Neustroev ${ }^{36}$, C. Nigro $^{5}$, K. Nilsson ${ }^{25}$, K. Nishijima ${ }^{32}$, K. Noda $^{6}$, S. Nozaki ${ }^{31}$, Y. Ohtani' ${ }^{6}$, T. Oka ${ }^{31}$, J. Otero-Santos ${ }^{1}$, S. Paiano ${ }^{3}$, M. Palatiello ${ }^{2}$, D. Paneque ${ }^{15}$, R. Paoletti ${ }^{13}$, J. M. Paredes ${ }^{18}$, L. Pavletić ${ }^{23}$, P. Peñil ${ }^{9}$, M. Persic ${ }^{2,50}$, M. Pihet ${ }^{15}$, P. G. Prada Moroni ${ }^{17}$, E. Prandini ${ }^{11}$, C. Priyadarshi ${ }^{5}$, I. Puljak ${ }^{29}$, W. Rhode ${ }^{7}$, M. Ribó ${ }^{18}$, J. Rico ${ }^{5}$, C. Righi ${ }^{3}$, A. Rugliancich ${ }^{17}$, N. Sahakyan ${ }^{28}$, T. Saito ${ }^{6}$, S. Sakurai ${ }^{6}$, K. Satalecka ${ }^{14}$, F. G. Saturni ${ }^{3}$, B. Schleicher ${ }^{24}$, K. Schmidt $^{7}$, T. Schweizer ${ }^{15}$, J. Sitarek ${ }^{12}$, I. Šnidarić ${ }^{37}$, D. Sobczynska ${ }^{12}$, A. Spolon ${ }^{11}$, A. Stamerra ${ }^{3}$, J. Strišković ${ }^{30}$, D. Strom ${ }^{15}$, M. Strzys ${ }^{6}$, Y. Suda ${ }^{27}$, T. Surić ${ }^{37}$, M. Takahashi ${ }^{6}$, R. Takeishi ${ }^{6}$, F. Tavecchio ${ }^{3}$, P. Temnikov ${ }^{34}$, T. Terzić ${ }^{23}$, M. Teshima ${ }^{15,6}$, L. Tosti ${ }^{38}$, S. Truzzi $^{13}$, A. Tutone ${ }^{3}$, S. Ubach ${ }^{26}$, J. van Scherpenberg ${ }^{15}$, G. Vanzo $^{1}$, M. Vazquez Acosta ${ }^{1}$, S. Ventura ${ }^{13}$, V. Verguilov ${ }^{34}$, C. F. Vigorito ${ }^{21}$, V. Vitale ${ }^{39}$, I. Vovk $^{6}$, M. Will ${ }^{15}$, C. Wunderlich ${ }^{13}$, T. Yamamoto $^{40}$, and D. Zarić 29

${ }^{1}$ Instituto de Astrofísica de Canarias and Dpto. de Astrofísica, Universidad de La Laguna, E-38200, La Laguna, Tenerife, Spain ${ }^{2}$ Università di Udine and INFN Trieste, I-33100 Udine, Italy ${ }^{3}$ National Institute for Astrophysics (INAF), I-00136 Rome, Italy ${ }^{4}$ ETH Zürich, CH-8093 Zürich, Switzerland ${ }^{5}$ Institut de Física d'Altes Energies (IFAE), The Barcelona Institute of Science and Technology (BIST), E-08193 Bellaterra (Barcelona), Spain ${ }^{6}$ Japanese MAGIC Group: Institute for Cosmic Ray Research (ICRR), The University of Tokyo, Kashiwa, 277-8582 Chiba, Japan ${ }^{7}$ Technische Universität Dortmund, D-44221 Dortmund, Germany ${ }^{8}$ Croatian MAGIC Group: University of Zagreb, Faculty of Electrical Engineering and Computing (FER), 10000 Zagreb, Croatia ${ }^{9}$ IPARCOS Institute and EMFTEL Department, Universidad Complutense de Madrid, E-28040 Madrid, Spain ${ }^{10}$ Centro Brasileiro de Pesquisas Físicas (CBPF), 22290-180 URCA, Rio de Janeiro (RJ), Brazil ${ }^{11}$ Università di Padova and INFN, I-35131 Padova, Italy ${ }^{12}$ University of Lodz, Faculty of Physics and Applied Informatics, Department of Astrophysics, 90-236 Lodz, Poland ${ }^{13}$ Università di Siena and INFN Pisa, I-53100 Siena, Italy ${ }^{14}$ Deutsches Elektronen-Synchrotron (DESY), D-15738 Zeuthen, Germany ${ }^{15}$ Max-Planck-Institut für Physik, D-80805 München, Germany ${ }^{16}$ Instituto de Astrofísica de Andalucía-CSIC, Glorieta de la Astronomía s/n, 18008, Granada, Spain ${ }^{17}$ Università di Pisa and INFN Pisa, I-56126 Pisa, Italy ${ }^{18}$ Universitat de Barcelona, ICCUB, IEEC-UB, E-08028 Barcelona, Spain ${ }^{19}$ Armenian MAGIC Group: A. Alikhanyan National Science Laboratory, 0036 Yerevan, Armenia ${ }^{20}$ Centro de Investigaciones Energéticas, Medioambientales y Tecnológicas, E-28040 Madrid, Spain ${ }^{21}$ INFN MAGIC Group: INFN Sezione di Torino and Università degli Studi di Torino, I-10125 Torino, Italy 22 INFN MAGIC Group: INFN Sezione di Bari and Dipartimento Interateneo di Fisica dell'Università e del Politecnico di Bari, I-70125 Bari, Italy ${ }^{23}$ Croatian MAGIC Group: University of Rijeka, Department of Physics, 51000 Rijeka, Croatia ${ }^{24}$ Universität Würzburg, D-97074 Würzburg, Germany ${ }^{25}$ Finnish MAGIC Group: Finnish Centre for Astronomy with ESO, University of Turku, FI-20014 Turku, Finland ${ }^{26}$ Departament de Física, and CERES-IEEC, Universitat Autònoma de Barcelona, E-08193 Bellaterra, Spain ${ }^{27}$ Japanese MAGIC Group: Physics Program, Graduate School of Advanced Science and Engineering, Hiroshima University, 739-8526 Hiroshima, Japan ${ }^{28}$ Armenian MAGIC Group: ICRANet-Armenia at NAS RA, 0019 Yerevan, Armenia ${ }^{29}$ Croatian MAGIC Group: University of Split, Faculty of Electrical Engineering, Mechanical Engineering and Naval Architecture (FESB), 21000 Split, Croatia ${ }^{30}$ Croatian MAGIC Group: Josip Juraj Strossmayer University of Osijek, Department of Physics, 31000 Osijek, Croatia ${ }^{31}$ Japanese MAGIC Group: Department of Physics, Kyoto University, 606-8502 Kyoto, Japan ${ }^{32}$ Japanese MAGIC Group: Department of Physics, Tokai University, Hiratsuka, 259-1292 Kanagawa, Japan ${ }^{33}$ Saha Institute of Nuclear Physics, HBNI, 1/AF Bidhannagar, Salt Lake, Sector-1, Kolkata 700064, India ${ }^{34}$ Inst. for Nucl. Research and Nucl. Energy, Bulgarian Academy of Sciences, BG-1784 Sofia, Bulgaria ${ }^{35}$ Japanese MAGIC Group: Department of Physics, Yamagata University, Yamagata 990-8560, Japan ${ }^{36}$ Finnish MAGIC Group: Astronomy Research Unit, University of Oulu, FI-90014 Oulu, Finland ${ }^{37}$ Croatian MAGIC Group: Ruđer Bošković Institute, 10000 Zagreb, Croatia ${ }^{38}$ INFN MAGIC Group: INFN Sezione di Perugia, I-06123 Perugia, Italy ${ }^{39}$ INFN MAGIC Group: INFN Roma Tor Vergata, I-00133 Roma, Italy ${ }^{40}$ Japanese MAGIC Group: Department of Physics, Konan University, Kobe, Hyogo 6588501, Japan ${ }^{41}$ also at International Center for Relativistic Astrophysics (ICRA), Rome, Italy ${ }^{42}$ now at Department for Physics and Technology, University of Bergen, NO-5020, Norway ${ }^{43}$ now at University of Innsbruck ${ }^{44}$ also at Port d'Informació Científica (PIC), E-08193 Bellaterra (Barcelona), Spain ${ }^{45}$ now at Ruhr-Universität Bochum, Fakultät für Physik und Astronomie, Astronomisches Institut (AIRUB), 44801 Bochum, Germany ${ }^{46}$ now at Department of Astronomy, University of California Berkeley, Berkeley CA $94720{ }^{47}$ also at Dipartimento di Fisica, Università di Trieste, I-34127 Trieste, Italy 49 now at Laboratoire d'Annecy de Physique des Particules (LAPP), CNRS-IN2P3, 74941 Annecy Cedex, France ${ }^{50}$ also at INAF Trieste and Dept. of Physics and Astronomy, University of 
Bologna, Bologna, Italy 